\title{
INFECÇÕES SEXUALMENTE TRANSMISSÍVEIS E PROMOÇÃO DE SAÚDE EM POPULAÇÃO DE ASSENTAMENTO EM UM MUNICÍPIO DO PONTAL DO TRIÂNGULO MINEIRO
}

\author{
Karine Rezende de Oliveira \\ Universidade Federal de Uberlândia, Ituiutaba - MG. \\ karinerezende@ufu.br \\ Nathalia Souza Lima \\ Universidade Federal de Uberlândia, Ituiutaba - MG. \\ nat_sou@hotmail.com \\ César Gómez Hernández \\ Universidade Federal do Triângulo Mineiro, Uberaba - MG. \\ cesar_cgh@hotmail.com \\ Sandro Prado Santos \\ Universidade Federal de Uberlândia, Ituiutaba - MG. \\ sandro.santos@ufu.br
}

\begin{abstract}
Resumo
Trata-se de um relato de experiência de promoção em saúde realizada com moradores de um assentamento da reforma agrária no município de Ituiutaba, Minas Gerais, com o objetivo de orientar e desmitificar temas que envolvem as sexualidades, métodos contraceptivos e Infecções Sexualmente Transmissíveis (IST), com moradores de um assentamento. Esta intervenção foi necessária, uma vez que áreas ocupadas por assentados geralmente não apresentam serviços básicos em saúde e assistência em âmbito educacional, refletindo desatenção à educação e saúde dessas populações. Apesar do avanço alcançado em relação a prevenção e tratamento IST persistem como um sério problema de saúde pública, tendo maior prevalência em populações socialmente vulneráveis, dentre elas a população rural. Participaram das atividades indivíduos com idade entre 14 e 60 anos, residentes do assentamento. Baseando-se em uma perspectiva emancipatória e respeitando os saberes da comunidade, as atividades ocorreram na forma de palestras e oficinas e ao final foi distribuído material informativo. Durante as atividades foi observado a predominância de muitas crenças e mitos sobre as temáticas abordadas. Conclui-se que o trabalho mostra a contínua necessidade de ações de políticas públicas efetivas para educação e saúde, principalmente para a população de assentados, muitas vezes marginalizados e sem orientações preventivas.
\end{abstract}

Palavras-chave: Educação em saúde; Infecções Sexualmente Transmissíveis; População Rural.

\section{SEXUALLY TRANSMISSIBLE INFECTIONS AND HEALTH PROMOTION IN SETTLEMENT POPULATION IN THE MUNICIPALITY OF PONTAL OF TRIANGULO MINEIRO}

\begin{abstract}
Cidadania em Ação: Revista de Extensão e Cultura, Florianópolis (SC), v.4, n.2, jul./dez. 2019.
\end{abstract}


This is an account of experience in health promotion carried out with residents of an agrarian reform settlement in the municipality of Ituiutaba, Minas Gerais, with the aim of orienting and demystifying issues involving sexualities, contraceptive methods and Sexually Transmitted Infections (STI) with residents of a settlement. This intervention was necessary since areas occupied by settlers usually do not present basic health services and educational assistance, reflecting inattention to the education and health of these populations. Despite advances in prevention and treatment, STI persist as a serious public health problem, with a higher prevalence among socially vulnerable populations, including the rural population. Participating in the activities were individuals aged between 14 and 60 years, residents of the settlement. Based on an emancipatory perspective and respecting the knowledge of the community, the activities took place in the form of lectures and workshops, and at the end, informative material was distributed. During the activities it was observed the predominance of many beliefs and myths about the topics addressed. The conclusion it's the work shows the continuous need for effective public policies for education and health, especially for the population of settlers, who are often marginalized and without preventive guidance.

Key words: Health Education. Sexually Transmitted Infections. Rural Population.

\section{INFECCIONES SEXUALMENTE TRANSMISIBLES Y PROMOCIÓN DE LA SALUD EN LA POBLACIÓN DEL ACUERDO EN UNA MUNICIPALIDAD DEL REGION DEL PONTAL DO TRIÂNGULO MINEIRO}

\section{Resumen}

Este es el relato de una experiencia en promoción de la salud realizada con vecinos de un asentamiento de reforma agraria en el municipio de Ituiutaba, Minas Gerais, con el objetivo de orientar y desmitificar tema relacionados con la sexualidad, los métodos anticonceptivos y las Infecciones de Transmissión Sexual (ITS), con residentes de un asentamiento. Esta intervención fue necesaria, ya que las áreas ocupadas por colonos generalmente carecen de servicios básicos de salud y asistencia educativa, lo que refleja una fala de atención a la educación y salud de estas poblaciones. A pesar de los avances en la prevención y el tratamiento de las ITS, siguen siendo un grave problema de salud pública, con una mayor prevalencia en poblacones socialmente vulnerables, incluida la población rural. En las actividades participaron personas con edades comprendidas entre los 14 y los 60 años, residentes del asentamiento. Desde una perspectiva emancipadora y respetando el conocimiento de la comunidad, las actividades se desarrollaron en fora de conferencias y al final se distribuyó material informativo. Durante las actividades se observó el predominio de muchas creencias y mitos sobre los temas abordados. Se concluye que el trabajo muestra la continua necesidad de acciones efectivas de política pública en educación y salud, especialmente para la población de colonos, muchas veces marginada y sin pautas preventivas.

Palabras clave: Educación para la salud. Infecciones de trasmisión sexual. Población rural.

Cidadania em Ação: Revista de Extensão e Cultura, Florianópolis (SC), v.4, n.2, jul./dez. 2019. 


\section{INTRODUÇÃO}

Atividades extensionistas possibilitam a formação do indivíduo como um profissional, o habilitando, cada vez mais, para atuar na produção e difusão do conhecimento necessário para superar as desigualdades sociais existentes no País. As ações de extensão são fundamentais para a interação da Universidade envolvendo as atividades de ensino, de pesquisa e extensão com as demandas da maioria da população.

O Movimento dos Trabalhadores Rurais Sem Terra (MST) iniciou no Brasil em 1979, em virtude de importantes transformações econômicas envolvendo a modernização agrícola e desocupação de áreas por estes trabalhadores (CALVACANTE; NOGUEIRA, 2008).

Considerando a realidade de comunidades como assentamentos da reforma agrária, existem relatos do pouco investimento feito em programas sociais, seja em atendimento ou orientação básica de profilaxia, que atendam especificamente população de assentados e acampados.

A epidemia do Vírus da Imunodeficiência Humana (HIV) e desenvolvimento da Síndrome da Imunodeficiência Adquirida (AIDS) representam um grande impacto social, sendo considerados um dos grandes problemas de saúde pública da atualidade. Muitas ações no plano socioeconômico e cultural são planejadas visando seu controle. Acredita-se que 15 a $30 \%$ das crianças nascidas de mães soropositivas para o HIV contraem o vírus na gestação, trabalho de parto, ou mesmo durante o aleitamento (BATISTA et al. 2013). Além disso, segundo o Boletim Epidemiológico de AIDS publicado pelo Ministério da Saúde (BRASIL, 2019) indivíduos com a faixa etária correspondente a 25 a 39 anos, ambos os sexos biológicos, compõe o grupo com maior concentração dos casos de aids no Brasil. Para as faixas etárias de 20 a 24 e de 25 a 29 anos, as taxas de detecção dos homens são quase quatro vezes maiores do que as taxas das mulheres. Deve-se acrescentar ainda ao percentual os indivíduos com 10 a13 anos, uma vez que o Programa Nacional de Doenças Sexualmente Transmissíveis (DST/AIDS) os inclui no grupo infantil (OLIVEIRA; 2009).

Estudo realizado em grupos de assentados mostram o desconhecimento da população em relação a transmissão e profilaxia das IST. Sendo assim, as atividades de educação em saúde são consideradas importante instrumentos para a garantia na melhoria das condições de vida. Existem relatos do pouco investimento feito em programas sociais, seja em atendimento ou orientação básica de profilaxia, que atendam especificamente a população de assentados e 
acampados. A oferta de serviços de caráter social assistencialista não chega a atender 32,6\% dos acampamentos e 23,7\% dos assentamentos do Brasil e apenas 56,8\% dos assentamentos e 45,7\% dos acampamentos possuem postos atendimento em saúde que oferecem atendimentos e programas de educação em saúde, próximos às suas áreas (BRASIL, 2001).

Intervenções realizadas em assentamentos rurais no Rio Grande do Sul mostraram fragilidades e despreparo no enfrentamento de situações que demandam ações preventivas e, portanto, domínio de informações em relação as IST. Esse fato se agrava no plano do treinamento da sexualidade, até mesmo em dificuldades de "negociação", com o próprio companheiro, de práticas seguras para a prevenção de doenças sexualmente transmissíveis e mesmo da anticoncepção (SOARES; LOPES, 2011).

Outra avaliação realizada em assentamento sobre a realidade e possibilidades de educação popular para jovens por meio da realização de oficina sobre IST e gravidez precoce, apresentou um resultado positivo quanto a participação dos indivíduos, inclusive dos jovens, mesmo que de uma forma ainda tímida mas marcante (LOBO; 2009).

É importante ressaltar que muitas vezes, as oficinas e palestras realizadas em assentamentos consideram como IST apenas HIV/AIDS. Esse fato reforça a necessidade de intervenções ativas e atuais nestas comunidades em especial aos adolescentes (FLEURI; VIEIRA, 2004).

Em resumo, apesar da extensa divulgação e desenvolvimento de campanhas relacionadas ás formas de prevenção das IST/AIDS feitas no Brasil, ainda se observa a predominância de comportamentos de risco na população, independente da faixa etária o que assinala uma ausência de associação entre o acesso à informação e a transformação do saber em práticas no dia-a-dia dos indivíduos. Sendo assim, para que essa ausência reduza, faz-se indispensável o acesso à informação efetiva para possibilitar a aquisição de conhecimentos adequados à promoção de sua saúde (OLIVEIRA; 2009).

A análise de trabalhos desenvolvidos a respeito da saúde dos moradores de assentamentos da reforma agrária mostra a ausência de intervenções de equipes da saúde dos municípios relacionadas à aspectos da saúde como sexualidade, uso de drogas, transmissão de doenças infecciosas dentre outros, nas comunidades.

O pouco investimento feito em programas sociais, seja em atendimento ou orientação básica de profilaxia, que atendam especificamente população de assentados e acampados é 
nítido e as atividades de educação em saúde são consideradas importantes instrumentos colaborar com a melhoria das condições de vida.

Em virtude da necessidade do desenvolvimento de ações de educação em saúde em assentamentos da reforma agrária o presente estudo teve como objetivo ministrar palestras e desenvolver oficinas para promover a educação no âmbito da saúde coletiva, incentivando práticas que conduzam a prevenção de IST pelos moradores do assentamento além de propor ações que possibilitem a interação entre os grupos e a construção do respeito mútuo com grupos específicos acometidos ou não pelas IST.

\section{MÉTODOS}

\section{PÚBLICO ALVO}

Foram convidados moradores da Associação Geral dos Trabalhadores Rurais Assentados na Fazenda Divisa, localizada em assentamento da reforma agrária, zona rural do município de Ituiutaba, Minas Gerais com um total de 143 pessoas associadas, ou 53 famílias. Este projeto foi aprovado pelo comitê de ética em pesquisa da Universidade Federal de Uberlândia, protocolo CAAE: 40768714.2.0000.5152. Inicialmente foram realizadas atividades envolvendo a equipe extensionista para avaliar aspectos metodológicos que possibilitariam a execução das ações dentro do contexto reforma agrária e assentamento rural.

Em seguida foram ministradas palestras para população do assentamento a qual foi dividida em dois grupos (adolescentes e adultos) para facilitar e adequar a linguagem das informações. O assunto das palestras foi Promoção da educação em saúde- IST abordados temas relacionados às diferentes IST, caracterização, formas de transmissão, comportamento de risco, profilaxia e tratamentos. Junto a equipe executora do projeto, uma profissional responsável pelo setor de acolhimento de pacientes portadores de doenças infecciosas da secretaria de saúde do município foi convidada para auxiliar na orientação quanto a prática segura do sexo e informar sobre os locais de auxílio e programas existentes para aconselhamento e acompanhamento psicológico e médico. Além disso, entre os temas das palestras discutiu-se a importância das IST em gestantes e pacientes imunodeprimidos, que apresentam alguma doença que favoreça a sua instalação e evolução. Durante a execução das palestras foi deixada uma caixa no local para coleta de perguntas sobre o tema, a fim de não 
constranger o participante. A caixa foi recolhida no final e as possíveis dúvidas sanadas.

Após a realização das palestras, a comunidade participou da oficina para Promoção da educação em saúde-IST com duração prevista de 4 horas para cada grupo (adolescentes e adultos). Foram reunidas pessoas com os mesmos interesses, considerando o assunto abordado, apresentada como natureza informativa, aperfeiçoando o que foi comentado nas palestras. Todos os participantes receberam cartilhas e folder com informações complementares dos assuntos abordados. Nestas oficinas os participantes confeccionaram material didático utilizando cartolinas, reportagens em revistas e jornais para afixar na sede do assentamento, uma vez que estes locais são frequentados por toda a comunidade do assentamento para realização de reuniões. Ao final da atividade cada grupo apresentou o seu material para a comunidade ressaltando algum aspecto importante relacionado a formas de contaminação e prevenção das IST.

\section{RESULTADOS}

Participaram deste estudo 43 moradores do Assentamento Divisa, zona Rural de Ituiutaba. A faixa etária predominante foi de 14 a 60 anos sendo a média da população de 49,1 anos $\pm 16,2$. As atividades foram realizadas durante os dias úteis da semana, assim, parte dos moradores da comunidade se encontrava fora do domicílio, exercendo atividades laborais inerentes a vida do campo, o que pode explicar o número de pessoas que compareceram após nosso convite.

Foi possível estabelecer um vínculo de confiança com a comunidade para o desenvolvimento das atividades propostas, criando uma concepção de saúde para jovens e adultos abrangendo o aspecto das IST. Além disso, conscientizar diferentes grupos etários da população acerca da importância clínica das IST, do uso de preservativos e a prática do sexo seguro por jovens e adultos a fim de evitar o contágio por alguma destas infecções. O estabelecimento do estigma e preconceito sofridos por indivíduos soropositivos para HIV, métodos contraceptivos, profilaxia, principais sintomas das ISTs e questões sociais envolvidas nas campanhas de prevenção também foram discutidos na roda de conversa.

As palestras direcionadas aos adolescentes ocorreram em momento diferente daquele dos adultos (muitas vezes, havia a presença dos pais) para não haver constrangimento.

A carência de informações sobre o assunto IST e assistência dada aos moradores da 
comunidade fez com que as atividades realizadas (palestras e oficinas) fossem executadas com dedicação e interesse.

Em relação às poucas perguntas deixadas pelos participantes nas caixas, todas foram respondidas, mas é importante ressaltar que a maioria dos questionamentos foi feito pelo próprio participante diante a comunidade durante a palestra, mostrando confiança com a equipe e valorização da atividade.

O material informacional (cartilha e folder) entregue foi considerado muito importante, pois alguns moradores relataram não haver conhecimento de nenhum dos assuntos abordados ou ao menos ter recebido algum material para esclarecimento durante a sua vida (Figura 1).

Com o apoio da Secretaria de Saúde do município, foram distribuídos preservativos aos presentes, com orientação da forma correta de uso.

Figura 1 - Material didático informativo distribuído para a comunidade após a finalização da oficina.

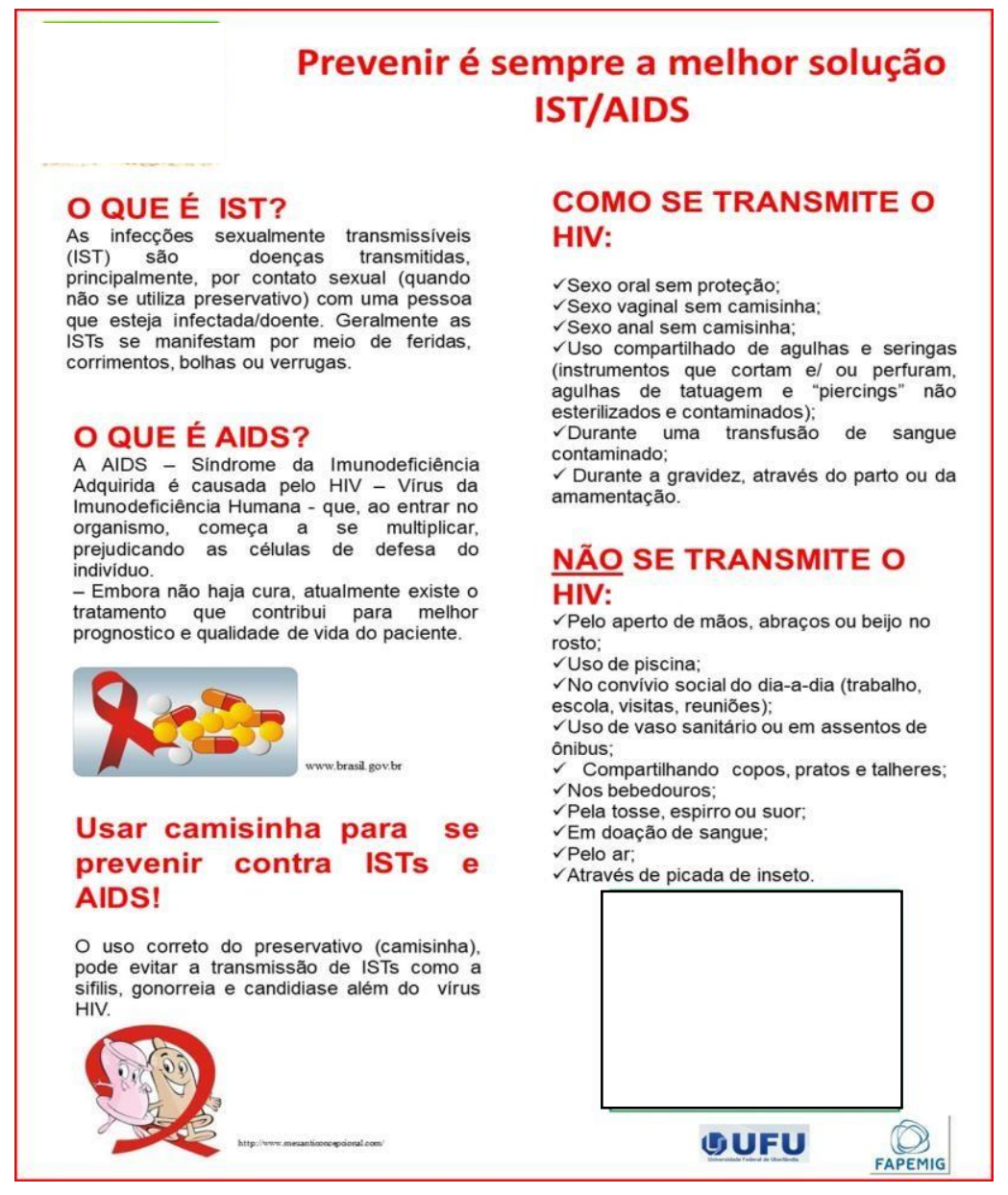

Fonte: Dos autores, 2019.

Cidadania em Ação: Revista de Extensão e Cultura, Florianópolis (SC), v.4, n.2, jul./dez. 2019. 
Em relação a confecção do material didático, os participantes consideraram muito interessante a atividade e a interação com os demais a tornou acolhedora e enriquecedora. $\mathrm{O}$ material feito continha orientações sobre as IST, como as formas de contaminação e comportamento de risco, sendo a maioria ressaltando a prevenção e necessidade de procurar ajuda médica, quando necessário.

\section{DISCUSSÃO}

A educação em saúde é definida como “[...] um conjunto de práticas pedagógicas de caráter participativo e emancipatório, que perpassa vários campos de atuação e tem como objetivo sensibilizar, conscientizar e mobilizar para o enfrentamento de situações individuais e coletivas que interferem na qualidade de vida.”. (BRASIL, 2009).

Muitas vezes, é importante destacar que ações envolvendo comunicação, informação, educação e escuta especializada favorecem a concretização da promoção da saúde e compreensão dos aspectos envolvidos em todo o contexto da sua criação e execução (BOEHS et al., 2007).

Segundo Scopinho (2010) até o ano 1999 não existiam diretrizes organizativas das ações de saúde no Movimento Sem Terra que pudessem envolver a política pública de saúde. Muitas vezes, a dificuldade de acesso aos serviços de saúde, como postos de atendimento ou médicos que poderiam visitar as comunidades de assentamentos da reforma agrária, tornaram o quadro da saúde nestes locais precários, aumentando os índices de morbidade, principalmente envolvendo IST. Além disso, observa-se a escassez de informação e cultura, o que torna a situação mais crítica (GODOY et al, 2000).

Em nossa experiência com a atividade extensionista na comunidade, observamos o grande interesse e a participação ativa de jovens, principalmente nas palestras. É importante relatar esse fato, uma vez que, segundo dados do Ministério da Saúde (BRASIL, 2011) no Brasil, do total de 608.230 casos registrados de AIDS, 66.114 deles são referentes a portadores jovens entre 13 a 24 anos. Este dado é muito relevante, considerando ser $11 \%$ dos casos notificados no país desde o início da epidemia (1980), com destaque para a transmissão sexual responsável por $68 \%$ dos casos.

Estudo realizado por Arraes et al. (2013) mostrou o quanto o grupo de jovens residentes em um assentamento na região do Brasil Central estão vulneráveis a contaminação, 
principalmente em decorrência da falta de informação e orientação. Ainda segundo as observações deste estudo, os jovens acreditam ser invulneráveis às doenças transmitidas pela via sexual associado a falta ou inexistência de conhecimento sobre as formas de prevenção às IST.

A interação dos participantes do grupo adulto foi intensa e muito produtiva, uma vez que a maioria relatou nunca ter participado de atividades ou recebido informações desta natureza.

Sabe-se que o número de novos casos de HIV/AIDS na população brasileira com idade acima de 50 anos aumento cerca de 3,6 para 7,1 em 100.000 habitantes no período compreendido entre 1996 a 2006. Este resultado mostra que houve aumento de 50\% na incidência da doença. Idoso portador desta doença apresenta grande relevância epidemiológica. Considerando os 47.437 casos de AIDS notificados desde o início da epidemia em 1980 em pessoas acima dos 50 anos, 29.393 (62\%) foram registrados de 2001 a junho de 2008. Ainda é importante ressaltar que nesse grupo de idosos, 37\% são mulheres e 63\% (PROGRAMA NACIONAL DST/AIDS, 2008; SOUZA et al., 2010)

Assim, intervenções como esta deveriam fazer parte da rotina da atenção primária desta comunidade, favorecendo a diminuição de casos de IST em virtude da falta de informação e prevenção.

As atividades de educação em saúde são consideradas importantes instrumentos para a garantia na melhoria das condições de vida. De acordo com a Organização Mundial de Saúde (OMS) o desenvolvimento de habilidades pessoais e coletivas contribui para a qualidade de vida e saúde da população. Para obter sucesso no processo educativo não devemos impor as informações e nem ao menos cobrar de forma ofensiva, mas de forma adequada às capacidades cognitivas de cada fase do desenvolvimento, num ambiente prazeroso, propiciando uma relação direta entre os conteúdos e o seu dia a dia, a contextualização do conhecimento (TOSCANI et al., 2007).

Segundo Arraes et al. (2013) as escolas e associação de moradores do assentamento deverão procurar apoio junto às instituições de assistência social do município para favorecer a realização de programas educativos para promoção da saúde sexual e reprodutiva do adolescente assentado.

Considerando os resultados observados nesta experiência extensionista, 152

Cidadania em Ação: Revista de Extensão e Cultura, Florianópolis (SC), v.4, n.2, jul./dez. 2019. 
acredita-se que este trabalho possa ser um importante indicador da necessidade de desenvolvimento de práticas educativas direcionadas para melhoria das condições de vida dos moradores do assentamento Divisa em relação as IST. Medidas profiláticas e a promoção da educação em saúde são imprescindíveis para manutenção da boa qualidade de vida considerando as IST e doenças associadas.

\section{AGRADECIMENTOS}

Fundação de Amparo à Pesquisa do Estado de Minas Gerais (FAPEMIG) e a Secretaria de Saúde, Prefeitura Municipal de Ituiutaba, Minas Gerais.

Cidadania em Ação: Revista de Extensão e Cultura, Florianópolis (SC), v.4, n.2, jul./dez. 2019. 


\section{REFERÊNCIAS}

ARRAES, C. O. et al. Masculinity, vulnerability and prevention of STD/HIV/AIDS among male adolescents: social representations in a land reform settlement. Revista Latinoamericana de Enfermagem, [s.1.], v. 21, n. 6, p.1266-1273, 2013.

BATISTA, M. G, et al. Conhecimento de mulheres acerca do HIV/AIDS: Realidade de um grupo de gestantes. Revista Ciência Saúde Nova Esperança, v.11, n.2, p. 10-9, 2013.

BOEHS, A. E. et al. Interface necessária entre enfermagem, educação e saúde e o conceito de cultura. Texto \& Contexto - Enfermagem, [s. 1], v.16, n.2, p. 307-314, 2007.

BRASIL. Ministério da Saúde. Saúde dos Trabalhadores Rurais de Assentamentos e Acampamentos da Reforma Agrária, 2001.

BRASIL. Ministério da saúde. Temática promoção da saúde IV. Brasília (DF): Organização Pan-Americana da Saúde; 2009.

CAVALCANTE, I. M. S.; NOGUEIRA, L. M. V. Práticas sociais coletivas para a saúde no assentamento Mártires de Abril na Ilha de Mosqueiro - Belém, Pará. Escola Anna Nery, [s.1.], v. 12, n. 3, p.492-499, 2008

FLEURI, R. M; VIEIRA, R. S. Juventude e sexualidade no contexto (Escolar) de assentamentos do movimento dos trabalhadores rurais sem terra. Dissertação (Mestrado em Educação) - Centro de Ciências da Educação, Universidade Federal de Santa Catarina, Florianópolis, 2004.

GODOY, A.; MORÁS, M.; AUCÉLIO, P. Q. A saúde dos trabalhadores rurais de assentamentos e acampamentos de reforma agrária. Brasília: UNBCeam-Nesp/MST-Setor Nacional de Saúde, 2000.

OLIVEIRA, D. C; GOMES, A. M. T; RIBEIRO, M. C. M; PONTES, A. P. M;

Conhecimentos E Práticas De Adolescentes Acerca das DST/HIV/AIDS em Duas Escolas Públicas Municipais do Rio de Janeiro. Escola Anna Nery Revista de Enfermagem, v.13, n.4, p. 833-841. 2009.

SCOPINHO, R. A. Condições de vida e saúde do trabalhador em assentamento rural. Ciência \& Saúde Coletiva, [s.1.], v. 15, n. 1, p.1575-1584, 2010.

SOARES, J. S. F; LOPES, M. J. M. Biografias de gravidez e maternidade na adolescência em assentamentos rurais no Rio Grande do Sul. Revista da Escola de Enfermagem, [s.1], v.45, n. 4, p.802-810, 2011. 
SOUZA, Z.; LEITE, J. L. Aids e envelhecimento: reflexões sobre a infecção pelo HIV em indivíduos acima dos 60 anos. Academus revista científica da saúde, Rio de Janeiro, v. 2, n. 2, 2003, 5p.

TOSCANI, N. V. et al. Desenvolvimento e análise de jogo educativo para crianças visando à prevenção de doenças parasitológicas. Interface - Comunicação, Saúde, Educação, [s.1] v. 11, n. 22, p. 281-294, 2007. 